\title{
Osteogenic activity of vanadyl(IV)-ascorbate complex: Evaluation of its mechanism of action
}

\author{
Ana M. Cortizo ${ }^{\mathrm{a}, *}$, M. Silvina Molinuevo ${ }^{\mathrm{a}}$, \\ Daniel A. Barrio ${ }^{a}$, Liliana Bruzzone ${ }^{b}$ \\ a Cátedra de Bioquímica Patológica, Facultad de Ciencias Exactas, Universidad Nacional de La Plata, \\ 47 y 115, 1900 La Plata, Argentina \\ b División Química Analítica, Facultad de Ciencias Exactas, Universidad Nacional de La Plata, \\ 47 y 115, 1900 La Plata, Argentina
}

Received 24 June 2005; received in revised form 16 November 2005; accepted 11 December 2005

Available online 9 January 2006

\begin{abstract}
We have previously shown that different vanadium(IV) complexes regulate osteoblastic growth. Since vanadium compounds are accumulated in vivo in bone, they may affect bone turnover. The development of vanadium complexes with different ligands could be an alternative strategy of use in skeletal tissue engineering. In this study, we have investigated the osteogenic properties of a vanadyl(IV)-ascorbate (VOAsc) complex, as well as its possible mechanisms of action, on two osteoblastic cell lines in culture. VOAsc $(2.5-25 \mu \mathrm{M})$ significantly stimulated osteoblastic proliferation $(113-125 \%$ basal, $p<0.01)$ in UMR106 cells, but not in the MC3T3E1 cell line. VOAsc $(5-100 \mu \mathrm{M})$ dose-dependently stimulated type-I collagen production (107-156\% basal) in osteoblasts. After 3 weeks of culture, 5-25 $\mu \mathrm{M}$ VOAsc increased the formation of nodules of mineralization in MC3T3E1 cells (7.7-20-fold control, $p<0.001)$. VOAsc $(50-100 \mu \mathrm{M})$ significantly stimulated apoptosis in both cell lines $(170-230 \%$ basal, $p<0.02-0.002)$, but did not affect reactive oxygen species production. The complex inhibited alkaline and neutral phosphatases from osteoblastic extracts with semi-maximal effect at $10 \mu \mathrm{M}$ doses. VOAsc induced the activation and redistribution of P-ERK in a time- and dose-dependent manner. Inhibitors of the mitogen activated protein kinases (MAPK) pathway (PD98059 and UO126) partially blocked the VOAsc-enhanced osteoblastic proliferation and collagen production. In addition, wortmanin, a PI-3-K inhibitor and type-L channel blocker nifedipine also partially abrogated these effects of VOAsc on osteoblasts. Our in vitro results suggest that this vanadyl(IV)-ascorbate complex could be a useful pharmacological tool for bone tissue regeneration.
\end{abstract}

(c) 2005 Elsevier Ltd. All rights reserved.

Keywords: Osteogenesis; Vanadium; Proliferation; Differentiation; Protein phosphorylation

Abbreviations: ALP, alkaline phosphatase; DHR, dihydrorhodamine123; DiFMUP, 6,8-difluoro-4-methylumbelliferyl phosphate; ERK, extracellular regulated kinases; $p$-NPP, para-nitro phenylphosphate; ROS, reactive oxygen species; VOAsc, vanadyl(IV)-ascorbate

* Corresponding author. Tel.: +54 221423 5333x49; fax: +542214223409.

E-mail address: cortizo@biol.unlp.edu.ar (A.M. Cortizo).

\section{Introduction}

Bone is a dynamic, highly vascularised tissue with a unique capacity to heal and remodel throughout life (Salgado, Coutinho, \& Reis, 2004). The integrity of the skeleton requires the coordinated regulation and activity of bone-forming cells (osteoblasts) and boneresorbing cells (osteoclasts). These cells are involved in the turnover of bone matrix components: hydroxyapatite 
and the organic part composed by glycoproteins, proteoglycans, sialoproteins, bone "gla" proteins. A number of growth factors and cytokines are produced in bone that regulate and stimulate osteogenesis and bone repairs (Tuan, 2004). The maintenance of a balance between formation and resorption results from opposing effects on the life span of osteoblasts and osteoclasts, including anti-apoptotic effects on the former and pro-apoptotic effect on the latter cell type (Kousteni et al., 2002; Manolagas, 2000). Thus, disbalance in skeletal cell activity can result in severe bone alterations. Agents used commonly for the treatment of metabolic bone diseases, such as parathyroid hormone and bisphosphonates, exert their beneficial effects on bone by regulating the rate of birth of new osteoblasts or osteoclasts or their apoptosis (Plotkin et al., 1999).

Vanadium compounds are insulin- and growth factormimetic compounds, which have been investigated by in vitro and in vivo studies (Srivastava \& Mehdi, 2005; Thompson \& Orvig, 2004). Vanadium salts stimulate glucose consumption, decreasing high blood sugar levels and insulin resistance of diabetic patients. In vivo studies have been carried out to investigate vanadium biodistribution. It has been shown that vanadium compounds are mainly localized in bone, as well as in kidney, liver and spleen after $24 \mathrm{~h}$ of administration. In particular, vanadium accumulated in the skeleton could exert specific effects that eventually may affect bone turnover.

In this context, our group has previously demonstrated that vanadate, vanadyl and different vanadium(IV) complex regulate proliferation and differentiation of osteoblast-like cells in culture (Barrio, Braziunas, Etcheverry, \& Cortizo, 1997; Barrio, Williams, Cortizo, \& Etcheverry, 2003; Etcheverry, Barrio, Cortizo, \& Williams, 2002; Etcheverry, Williams et al., 2002). In general, low concentrations $(<25 \mu \mathrm{M})$ stimulate but higher doses inhibit cell proliferation and alkaline phosphatase activity (ALP), resulting in cytotoxic effects on osteoblasts. Several mechanisms have been proposed to explain the bioactivity of vanadium. The best characterized action is its ability to stimulate the protein tyrosine phosphorylation through the inhibition of protein tyrosine phosphatases (PTPases) (Tracey \& Gresser, 1986). As a consequence, a phosphorylation cascade can be triggered. For example, the mitogen activated protein kinases (MAPK) pathway and extracellular signal-regulated kinase (ERK) activation have been reported to be involved in the action of vanadium (Barrio et al., 2003; Shechter et al., 1995). It is known that ERK plays an important role in osteoblast function, including the regulation of osteoblast-specific gene expression, proliferation, matrix mineralization, and adhesion (Lai et al., 2001; Manolagas, 2000; Xiao, Jiang, Gopalakrishnan, \& Franceschi, 2000). Apoptosis is an active process of cell death that could be involved in the regulation of bone turnover under physiological or pathological conditions. We have recently shown that several vanadium compounds stimulate apoptosis by mechanisms which involve induction of intracellular oxidative stress (Molinuevo, Barrio, Cortizo, \& Etcheverry, 2004). Thus, under certain conditions, vanadium compounds could exert toxic effect, depending on several factors (Domingo, 2002). It is important to evaluate the possible cytotoxic effects of new vanadium compounds before their can be considered for use in vivo.

Until the present, no vanadium compounds with specific properties to induce osteogenesis have been described. In this study, we have investigated the osteogenic properties of a vanadyl(IV)-ascorbate (VOAsc) complex, as well as its possible mechanisms of action on two osteoblast-like cells in culture.

\section{Materials and methods}

\subsection{Materials}

Dulbecco's modified Eagle's medium (DMEM), trypsin-EDTA and fetal bovine serum (FBS) were from Gibco (Life Technology, Buenos Aires, Argentina) and tissue culture disposable material was from Nunc (Buenos Aires, Argentina). Dihydrorhodamine123 (DHR), annexin V-FITC and propidium iodide (PI) were from Molecular Probes (Eugene, OR, USA). Goat polyclonal anti-ERK-1/2 antibodies, as well as a monoclonal anti-P-ERK antibody, were from Santa Cruz Biotechnology Inc. (Santa Cruz, CA, USA). All other chemicals and reagents were obtained from commercial sources and were of analytical grade. Vanadyl(IV)-ascorbate was synthesized as previously described by Ferrer and characterized by UV-vis and infrared spectroscopy (Ferrer, Williams, \& Baran, 1998). Stock solutions were prepared in distilled water at room temperature and immediately used in the different assays.

\subsection{Cell culture and incubations}

UMR106 rat osteosarcoma cells and MC3T3E1 mouse calvaria-derived cells were grown in DMEM containing $10 \% \mathrm{FBS}, 100 \mathrm{U} / \mathrm{ml}$ penicillin and $100 \mu \mathrm{g} / \mathrm{ml}$ streptomycin at $37^{\circ} \mathrm{C}$ in $5 \% \quad \mathrm{CO}_{2}$ atmosphere (McCarthy, Etcheverry, Bruzzone, \& Cortizo, 1997). Cells were seeded on $75 \mathrm{~cm}^{2}$ flasks and sub-cultured using trypsin-EDTA and replated on multi-well plates. 
The UMR106 cell line has been shown to conserve certain characteristics of differentiated osteoblastic phenotype (Partridge, Alcorn, Michelangeli, Ryan, \& Martin, 1983). In the case of non-transformed MC3T3E1 cells, previous studies have demonstrated that expression of osteoblastic markers begins after culturing the cells with medium supplemented by $5 \mathrm{mM} \beta$-glycerol phosphate ( $\beta \mathrm{GP}$ ) and $145 \mu \mathrm{M}$ ascorbic acid (AA) (Quarles, Yohay, Lever, Caton, \& Wenstrup, 1992). Under these culture conditions, alkaline phosphatase activity begins to be expressed after 10 days, and mineralisation is achieved after extending the culture to 15 days. However, the cells only undergo active replication during the first 5 days of incubation.

For proliferation experiments with both cell lines, and differentiation experiments with UMR106 osteoblasts, cells on multi-well plates were incubated in serumfree medium with different doses of VOAsc complex solution (with or without inhibitors) during the periods of time indicated in the legend of figures. For ALP and type-I collagen production experiments with MC3T3E1 osteoblasts, cells were cultured for 2 weeks in DMEM-10\% FBS supplemented with $\beta$ GP and AA, changing the medium every other day, after which they were serum-starved and incubated in DMEM with different doses of VOAsc complex solution for an additional $48 \mathrm{~h}$. For mineralization experiments with MC3T3E1 osteoblasts, cells were cultures for 2 weeks in DMEM-10\% FBS supplemented with $\beta$ GP and AA plus different concentrations of VOAsc complex solution, changing this complete medium every 2 days.

\subsection{Cell proliferation assay}

Cell proliferation was determined using the crystal violet bioassay as we have previously described (Molinuevo et al., 2004).

\subsection{Assays for osteoblast differentiation and maturation}

Osteoblastic differentiation was evaluated by two markers: alkaline phosphatase activity, as we have previously described (Molinuevo et al., 2004) and the quantitation of type-I collagen production with a Sirius red-based colorimetric microssay (Tullberg-Reinert \& Jundt, 1999). For the latter, cells were fixed in Bouin's fluid for $1 \mathrm{~h}$, washed with water and stained with Sirius red dye for $1 \mathrm{~h}$. The stained material was dissolved in $0.1 \mathrm{~N}$ sodium hydroxide and the absorbance read at $550 \mathrm{~nm}$.
Mineralized nodules in the extracellular matrix of MC3T3E1 osteoblasts were stained with Alizarin red S and quantified by the measurement of the soluble calcium deposition (Ueno, Kitase, Moriyama, \& Inoue, 2001).

\subsection{Evaluation of cell death}

The mechanism of cell death induced by VOAsc was evaluated using an annexin V-FITC/propidium iodide assay as we have previously described (Molinuevo et al., 2004). Early apoptotic stages were characterized by annexin V-FITC-positive/PI-negative $\left(\mathrm{V}^{+} / \mathrm{PI}^{-}\right)$staining. The cell number was determined by counting the cells per field in eight fields per coverslips.

\subsection{Determination of reactive oxygen species (ROS) production}

The possible VOAsc-induced oxidative stress in osteoblasts was evaluated by measurement of intracellular production of reactive oxygen species. ROS generation was measured by oxidation of DHR to rhodamine as we have previously described (Cortizo, Bruzzone, Molinuevo, \& Etcheverry, 2000).

\subsection{Phosphatase assay}

A kinetic assay was performed using a $0.1 \%$ Triton$\mathrm{X} 100$ osteoblast extract and the fluorogenic small substrate 6,8-difluoro-4-methylumbelliferyl phosphate (DiFMUP) (Peters et al., 2003). The cell extract was incubated with $10 \mu \mathrm{M}$ DiFMUP in phosphate buffer, $\mathrm{pH}$ 7.0 , for $15 \mathrm{~min}$. The resulting product was measured at $360 / 450 \mathrm{~nm}$ (excitation/emission wavelengths) using an Aminco-Bowman SPF100 spectrofluorometer. Different concentrations of VOAsc were pre-incubated with the osteoblastic extract for $10 \mathrm{~min}$ before substrate addition.

\subsection{Western blot analysis of P-ERK}

We next evaluated the activation status of the ERK$1 / 2$ transduction pathway induced by VOAsc, using a method previously described (Barrio et al., 2003). In these experiments, osteoblastic cells growing in serumfree medium with the addition of different concentrations of VOAsc, were lysated in Laemmli's buffer (Laemmli, Beguin, \& Gujer-Kellenberger, 1979) and the protein content was evaluated by the method of Lowry, Rosebrough, Farr, and Randall (1951). These lysates were heated at $100^{\circ} \mathrm{C}$ for $3 \mathrm{~min}$ and aliquots corresponding to $30 \mu \mathrm{g}$ of total protein were subjected 
to $12 \%$ SDS-PAGE. The separated proteins were then electro-transferred to nitrocellulose membranes. After washing and blocking, the membranes were incubated with a monoclonal anti-P-ERK antibody, or a polyclonal antibody that recognizes both phosphorylated and unphosphorylated ERK-1/2. Blots were developed using chemioluminiscence reagents. The intensity of the specific bands was quantified by densitometry after scanning of the photographic film. Images were analysed using the Scion-beta 2 program.

\subsection{Localization of P-ERK by immunofluorescence analysis}

Cells grown on coverslips were fixed in $4 \%$ $p$-formaldehyde in phosphate-buffered saline (PBS), blocked and incubated with a monoclonal anti-P-ERK antibody $(1: 200)$ at $4{ }^{\circ} \mathrm{C}$ for $16 \mathrm{~h}$. After washing, a secondary FITC-goat anti-mouse antibody (1:200) was added for $2 \mathrm{~h}$ at room temperature. Coverslips were mounted in a Vectastain mounting liquid and the distribution of the specific protein was imaged using a Nikon-5000 fluorescence microscope and a digital camera.

\subsection{Stability studies}

To test the stability of VOAsc solutions under the different experimental conditions used in this work, we used a method previously described by our group (Barrio et al., 2003; Williams, Molinuevo, Okulik, Jubert, \& Etcheverry, 2005). The rates of the decomposition reaction of VOAsc complex in water, culture medium or glycine buffer, $\mathrm{pH} 10$, were determined by measuring the time-dependent variation of the UV-vis spectra at 25 or $37^{\circ} \mathrm{C}$. In order to prevent contact of the sample with
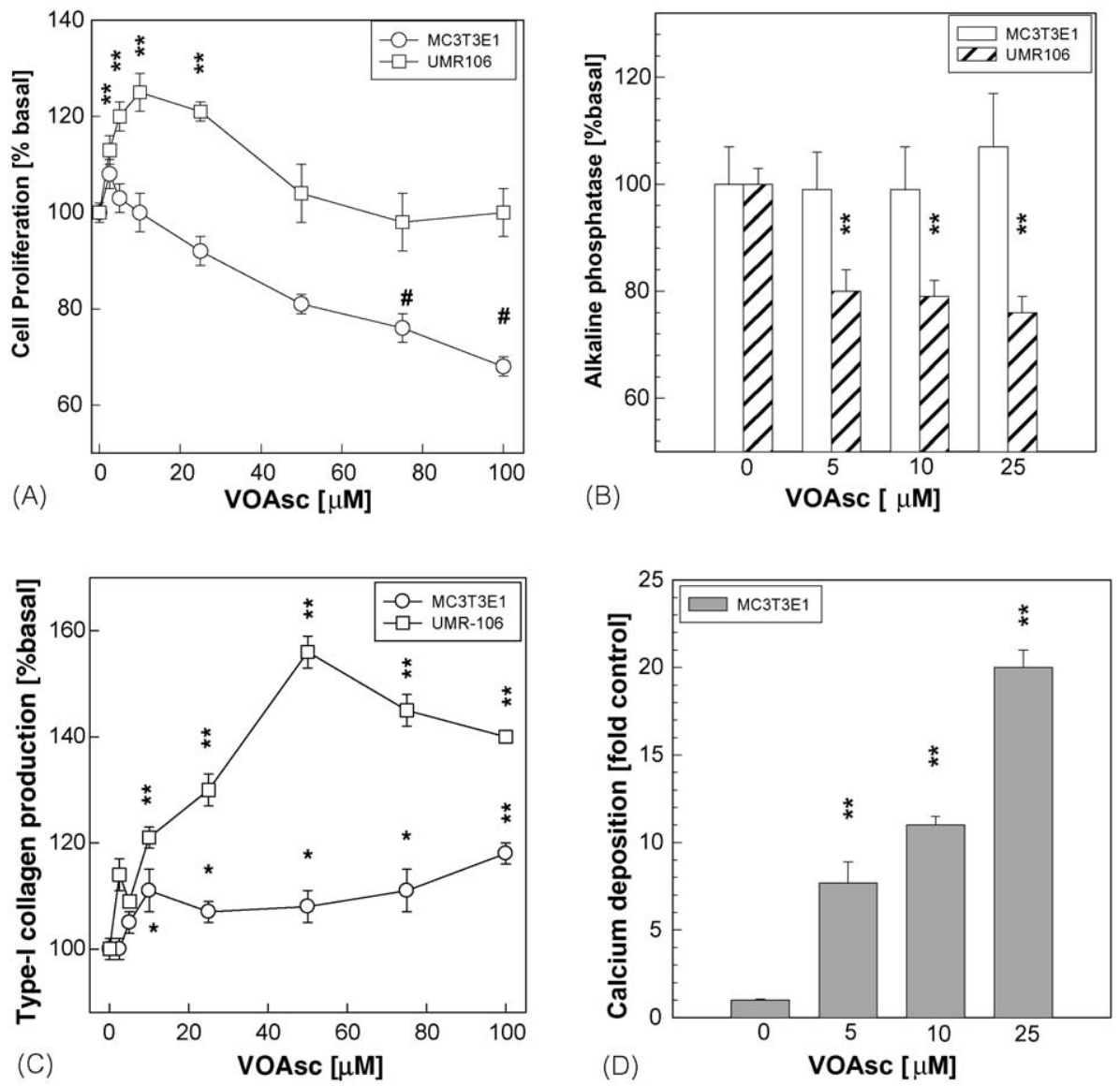

Fig. 1. Effect of VOAsc on cell proliferation, differentiation and mineralization. MC3T3E1 and UMR106 cells were incubated with different doses of VOAsc. Cell proliferation was determined by crystal violet assay after $24 \mathrm{~h}$ of culture (A), osteoblastic differentiation was assessed by alkaline phosphatase activity (B), type-I collagen production (C) after $48 \mathrm{~h}$ of culture, and matrix mineralization of MC3T3E1 cells was evaluated after 2 weeks of culture by calcium nodules staining with Alizarin red (D). Data represent the mean \pm S.E.M. of three independent experiments and are expressed as $\%$ basal or fold control $\left({ }^{\#} p<0.05,{ }^{*} p<0.01\right.$ and $\left.{ }^{* *} p<0.001\right)$. 
atmospheric oxygen, the measurements were performed directly in the spectrophotometer cell, adequately covered with a stopped and parafilm.

\subsection{Statistical analysis}

Three independent experiments were run for each experimental condition. Results are expressed as the mean \pm S.E.M. Statistical analysis of the data was performed by Student's $t$-test.

\section{Results}

\subsection{Effects of VOAsc on proliferation, differentiation and mineralization of osteoblast-like cells}

In order to investigate the effect of VOAsc on cell proliferation and differentiation, both osteoblast-like cell lines (MC3T3E1 and UMR106) were used. Treatment of osteoblasts with VOAsc $(2.5-100 \mu \mathrm{M})$ for $24-48 \mathrm{~h}$ led to a biphasic effect on cell proliferation as determined by the crystal violet bioassay. Fig. 1A shows that low doses of VOAsc $(2.5-25 \mu \mathrm{M})$ significantly stimulated UMR106 osteoblast proliferation, while higher doses did not affect (UMR106) or inhibited (MC3T3E1) cell growth. To examine the effect of VOAsc on osteoblast differentiation, alkaline phosphatase activity and type-I collagen production were evaluated in both cell lines. As can be seen in Fig. 1B, the vanadium compound $(5-25 \mu \mathrm{M})$ inhibited ALP in UMR106 cells although it did not affect this marker of differentiation in the MC3T3E1 pre-osteoblasts. Under similar conditions, VOAsc dose-dependently stimulated the formation of a collagenous matrix both in the UMR106 and the MC3T3E1 cell line (Fig. 1C). Furthermore, the effect of this vanadium complex on the ability of MC3T3E1 cells to mineralize the matrix was assessed after 2 weeks of culture in the presence of $\beta$-glycerol phosphate and ascorbic acid. Fig. 1D shows that VOAsc markedly and dose-dependently stimulated calcium deposition in mineralized nodules as assessed by the Alizarin red S staining. These results indicate that at low doses $(<25 \mu \mathrm{M})$, VOAsc is a weak mitogen and, although it partially inhibits ALP, its long-term exposure to osteoblastic cultures ( 2 weeks) results in osteogenic effects.

\subsection{Mechanism of cell death and oxidative stress induced by VOAsc}

To evaluate the possible effect of VOAsc on cell death, early apoptotic processes were analyzed by annexin-

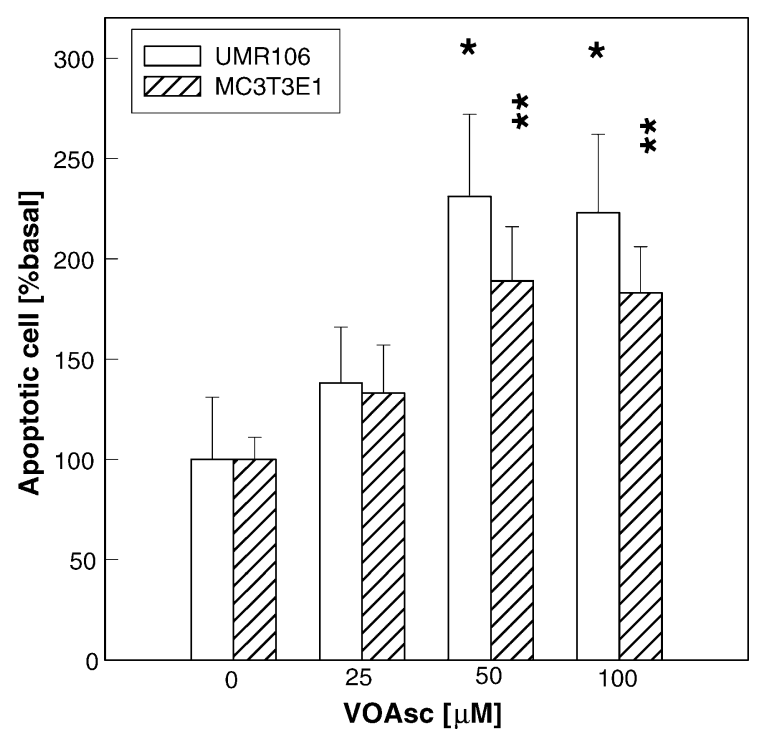

Fig. 2. Effect of VOAsc on apoptosis. Osteoblasts were treated with 0-100 $\mu \mathrm{M}$ VOAsc for $4 \mathrm{~h}$. Apoptosis was assessed by annexin V/PI staining as it was described in Section 2. Data are expressed as \% basal and represent the mean \pm S.E.M. of three experiments $\left({ }^{*} p<0.02\right.$ and $\left.{ }^{* *} p<0.01\right)$.

V/PI staining. Both cell lines were treated with the vanadium compound $(25-100 \mu \mathrm{M})$ for $4 \mathrm{~h}$. The results (Fig. 2) show that $25 \mu \mathrm{M}$ concentration did not induce cell death but higher concentrations $(50-100 \mu \mathrm{M})$ significantly stimulated apoptosis in both cell lines. Since these effects could be associated with induction of oxidative stress, we next investigated the ROS production after incubation with VOAsc. The vanadium compound $(25-100 \mu \mathrm{M})$ did not affect ROS production in the UMR106 osteoblastic cell line as evaluated by the DHR assay (data not shown). These data suggest that the VOAsc-induced apoptotic effect on osteoblastic cultures is not due to induction of oxidative stress.

\subsection{VOAsc inhibition of phosphatases}

Because vanadate and other vanadium complexes are potent inhibitors of different phosphatases, we investigated the possible inhibitory effect of VOAsc on alkaline and neutral phosphatases. For this purpose, an extract of osteoblastic cells was used as source of enzyme and incubated $10 \mathrm{~min}$ with the vanadium complex. When para-nitro phenylphosphate ( $p$-NPP) in a pH 10 buffer was used as substrate to evaluate ALP, VOAsc had a significant inhibitory effect with a dose-dependent pattern (Fig. 3). Maximal inhibition occurred at $100 \mu \mathrm{M}$ concentration with a half-effect at $10 \mu \mathrm{M}$. Likewise, by using the fluorogenic assay of DiFMUP substrate under neutral conditions to evaluate PTPases, an inhibitory effect was 


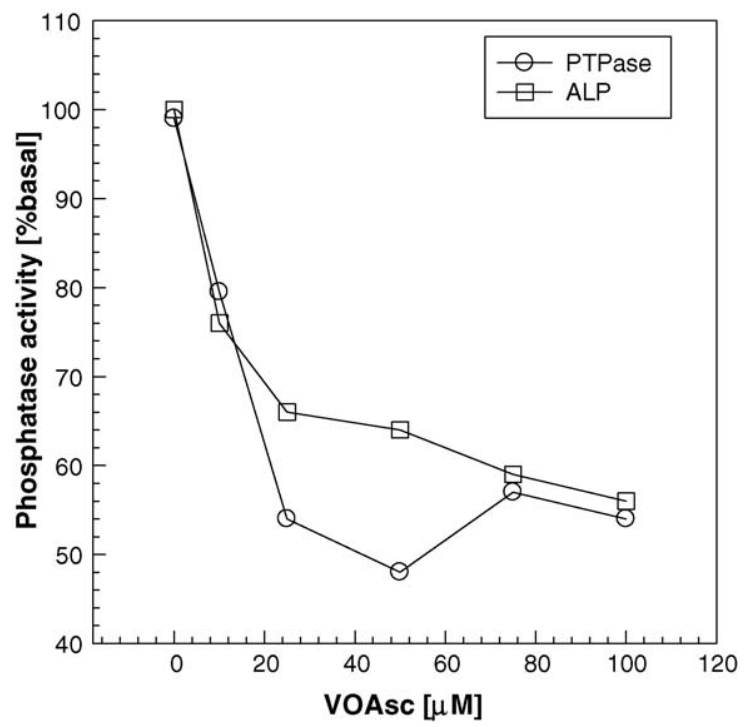

Fig. 3. Effect of VOAsc on phosphatase activity. UMR106 cellular extracts were incubated with $p$-NPP (ALP activity) or DiFMUP (PTPases) in the presence of different concentrations of VOAsc. Results are presented as \% basal (absence of VOAsc) and are representative of three experiments.

also observed in the range of concentrations in which VOAsc affects cell growth and differentiation. In this case, $50 \mu \mathrm{M}$ VOAsc induced the maximal inhibition with a semi-maximal effect at $10 \mu \mathrm{M}$.

\subsection{Activation and subcellular re-distribution of P-ERK induced by VOAsc}

To determine the role of MAPK (an important signaling intermediate of growth factor pathway) in the osteoblastic effects of VOAsc, we assessed the phosphorylation status of ERK-1/2. Treatment of osteoblasts with $1 \mathrm{mM}$ VOAsc resulted in a time-dependent increase of phosphorylated p44/42-ERK as evaluated using a phospho-ERK-specific antibody. This effect was also dependent on the concentration of the complex. VOAscinduced activation was most prominent after $1 \mathrm{~h}$ incubation (Fig. 4A) with a maximal phosphorylation increase at a concentration of $1 \mathrm{mM}$ (Fig. 4B). There was no change in protein amounts of ERK as assessed by immunoblots using anti-ERK antibodies (data not shown).

Because phosphorylation/activation of ERK depends on the presence of MAPK in the nucleus, we examined whether or not VOAsc alters the subcellular distribution of P-ERK. In control UMR106 osteoblasts, P-ERK was weakly detected in the cytoplasm with a diffuse and punctate pattern throughout the cell body (Fig. 5). Treat-
(A)
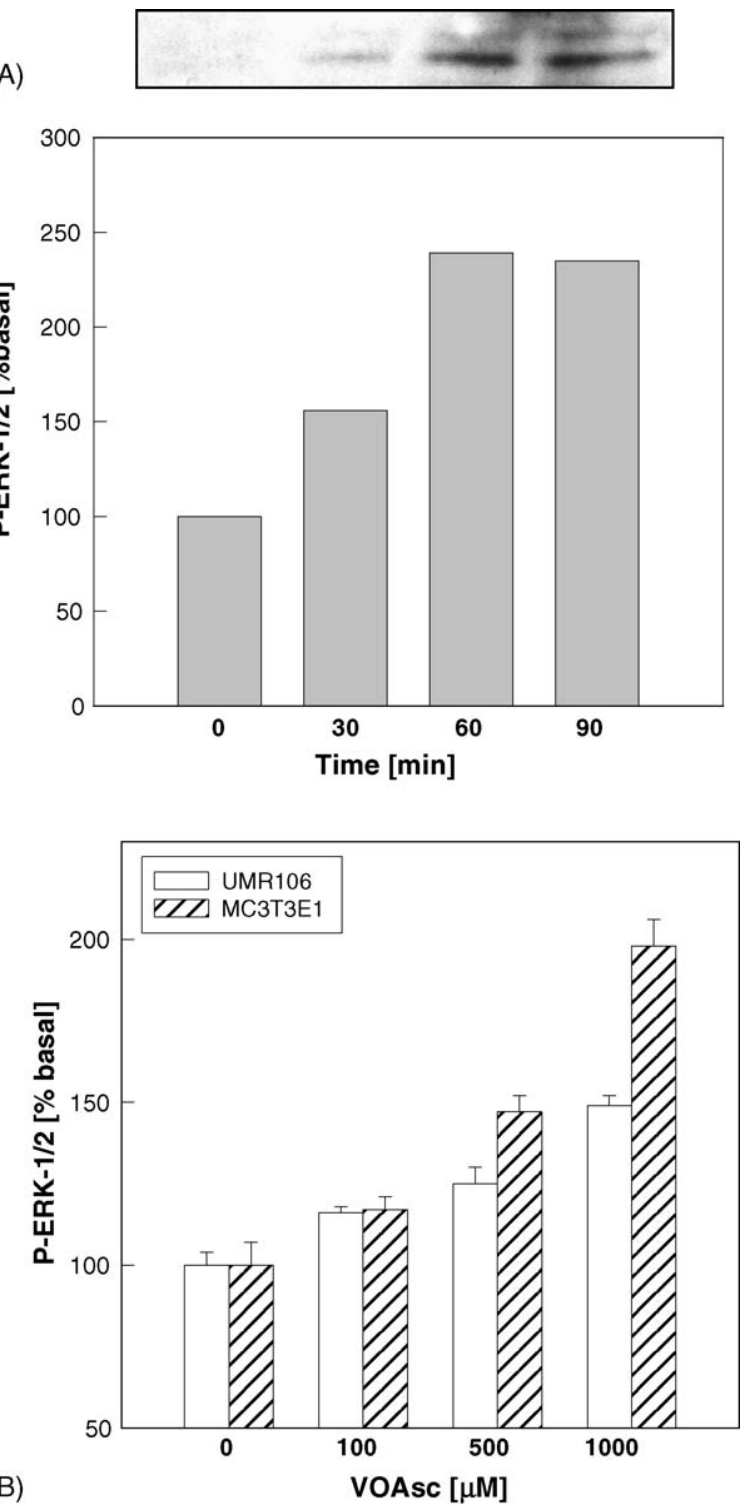

Fig. 4. Time and dose-dependent phosphorylation of ERK induced by VOAsc. Osteoblasts were treated with $1 \mathrm{mM}$ VOAsc for different periods of time (A) or incubated for $1 \mathrm{~h}$ with increasing doses of VOAsc (B). Cells were lysated in Laemmli's buffer and analyzed by Western blot using an anti-P-ERK antibody. Results are expressed as \% basal and represent the mean \pm S.E.M. of three independent experiments.

ment with $1 \mathrm{mM}$ VOAsc stimulated P-ERK-associated immunofluorescence after $15 \mathrm{~min}$, reaching a maximum by $30 \mathrm{~min}$ and persisting for $30-60 \mathrm{~min}$. This time course closely matched osteoblast P-ERK activation as detected by Western blot. In addition, P-ERK-associated fluorescence exhibited a re-distribution from the cytoplasm to the nucleus after $15-30 \mathrm{~min}$ incubation with $1 \mathrm{mM}$ VOAsc. The signal diminished and the remaining P-ERK was mainly cytoplasmic after $60 \mathrm{~min}$ incubation. These 

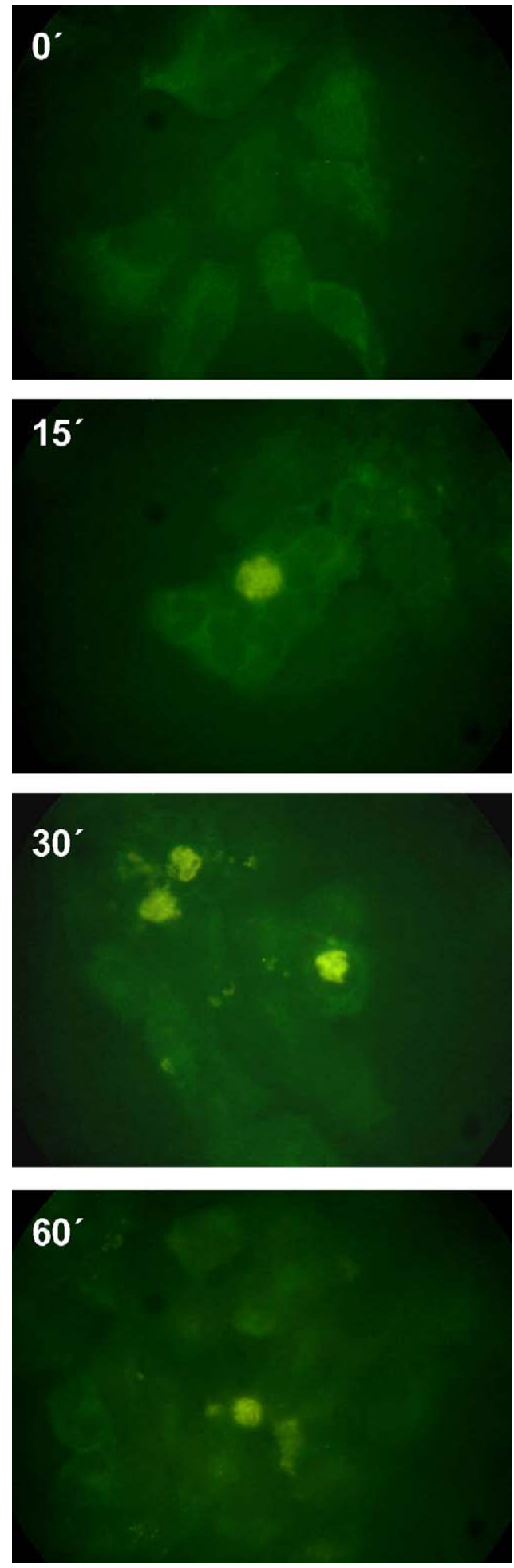

Fig. 5. VOAsc induced subcellular redistribution of P-ERK. UMR106 cells were treated with $1 \mathrm{mM}$ VOAsc for different periods of time and the P-ERK-associated inmunofluorescence was analyzed. Objective $40 \times$. results suggest that this vanadium complex could be regulating osteoblastic growth by the MAPK pathway.

\subsection{Signal transduction pathways involved in the osteogenic action of VOAsc}

In order to investigate the relative importance of different signal transduction pathways involved in the VOAsc-induced osteogenic actions, the effect of various
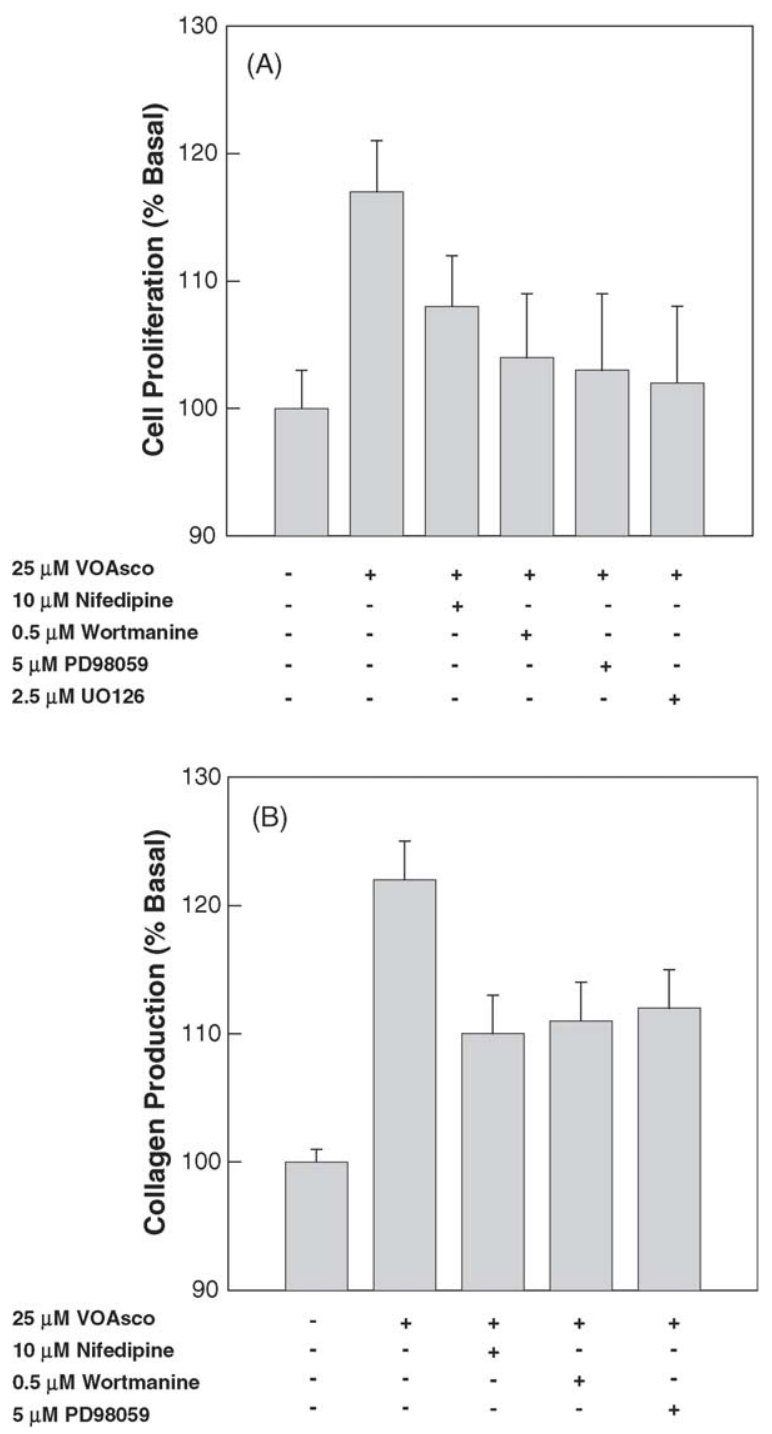

Fig. 6. Effect of different signalling pathways inhibitors on cell proliferation (A) and collagen production (B) induced by VOAsc. Cell cultures were incubated with $25 \mu \mathrm{M}$ VOAsc in the presence of nifedipine, wortmanin, PD98059 and UO126 during $24 \mathrm{~h}$. After this incubation period, cell proliferation or type-I collagen production assays were performed as it was described in Section 2. Data are expressed as mean \pm S.E.M. and are expressed as $\%$ basal of three independent experiments. 
inhibitors was evaluated (Fig. 6). We found that inhibition with PD98059 or UO126 (MAPK inhibitors) completely blocked VOAsc-enhanced proliferation (Fig. 6A) and about $50 \%$ of collagen production (Fig. 6B). In addition, wortmanin (a PI-3-K inhibitor) and the L-type calcium channel blocker nifedipine, also partly abolished the effects of the vanadium complex on proliferation and collagen production.

\subsection{Stability of VOAsc complex solutions}

The decomposition reactions of VOAsc were measured at $25^{\circ} \mathrm{C}$ since the stock solutions were prepared at room temperature, and at $37^{\circ} \mathrm{C}$, which is the temperature for biological assays. Plots of $\ln A$ versus time showed a linear decay of absorbance. The rate constant for the decomposition of VOAsc solution in distilled water at $25^{\circ} \mathrm{C}$ was $k=2.25 \times 10^{-4} \mathrm{~min}^{-1}$. On the other hand, in DMEM media at $37^{\circ} \mathrm{C}$, the $k$ value was $8.8 \times 10^{-4} \mathrm{~min}^{-1}$. These results suggest that VOAsc is stable under our working conditions, although its decomposition is greater in the culture media. In addition, we evaluated the stability of VOAsc complex in glycine buffer $\mathrm{pH} 10$ at $37^{\circ} \mathrm{C}$, the condition of the ALP assay. The rate constant for the decomposition was $k=0.8 \times 10^{-4} \mathrm{~min}^{-1}$. Considering that the ALP assay involved a short incubation period $(10 \mathrm{~min})$, these results suggest that VOAsc complex solution is very stable in the conditions of the phosphatase assay.

\section{Discussion}

In this study, we investigated the effect of a previously synthesized and characterized vanadium(IV) complex with ascorbic acid obtained at $\mathrm{pH}$ 7.0. Our present results demonstrate that VOAsc exerts osteogenic actions on osteoblasts in culture. Evidence for this notion is based in several observations: (a) low doses of VOAsc act as a weak mitogen stimulating osteoblastic proliferation, (b) although VOAsc partially inhibited ALP in the UMR106 osteosarcoma line, it did not affect this marker in the non-transformed MC3T3E1 cells, (c) VOAsc dose-dependently stimulated type-I collagen production in both cell lines and (d) after 2 weeks of culture, VOAsc strongly induced the formation of nodules of mineralization in MC3T3E1 cells, which is a much shorter period of time than 3-4 weeks of culture required for mineralization of these cells which has been previously described (Quarles et al., 1992). Thus, this vanadium compound not only induced growth and differentiation but also had the potential to increase mineralization of the matrix, which is an important osteoblastic function.
We have previously reported that other vanadium(IV) compounds affected osteoblast proliferation and differentiation in a biphasic manner (Barrio et al., 1997; Barrio et al., 2003; Etcheverry, Barrio et al., 2002; Etcheverry, Williams et al., 2002). For example, complexes with maltol, aspirin, other anti-inflamatory compounds and the sugar threalose at low doses stimulated cell growth, but inhibited ALP-associated osteoblastic differentiation. In addition, high concentrations and/or long-term exposure to vanadium complexes showed cytotoxic effect on osteoblastic cultures. Since vanadium compounds could have potential clinical applications, it is relevant to evaluate their possible toxicity (Domingo, 2002).

In the present study, we found that incubation with ascorbic acid alone did not affect osteoblastic proliferation, ALP-specific activity nor type-I collagen production (data not shown). The importance of ascorbic acid in the process of osteoblastic differentiation and mineralization is well known. The osteogenic effects reported for VOAsc were observed at doses of 2.5-25 $\mu \mathrm{M}$, which are at least 6-60-fold lower than the ascorbic acid concentration used in the mineralization induction medium $(145 \mu \mathrm{M})$. Recently, Park et al. (2004) reported that ascorbic acid inhibited cell proliferation and induced apoptosis in leukemia cells via hydrogen peroxidemediated mechanisms. These effects were detected at concentrations of $0.25-1.0 \mathrm{mM}$, which are much higher doses than those used in our present study. They also observed that the same concentrations of ascorbic acid had no significant effect on three ovarian cancer cell lines, suggesting that the cytotoxic effect of ascorbic acid was cell-type specific.

Apoptosis is an important process that controls cell death and tissue regeneration (Steller, 1995). It has been suggested that oxidative stress triggers signaling mechanisms that finally lead to apoptosis. The same growth factors and cytokines that stimulate osteoclast and osteoblast development can also influence their apoptosis (Manolagas, 2000). In the present study, low doses of VOAsc $(<25 \mu \mathrm{M})$ did not induce apoptosis in osteoblastic cells. However, this compound effectively enhanced apoptosis at higher concentrations $(50-100 \mu \mathrm{M})$. This effect was apparently not associated with oxidative stress since no increase in ROS production was detected in cells treated with high concentrations of VOAsc, as assessed by the DHR assay. We also evaluated the possible effect of VOAsc on lipid peroxidation by TBARS production, however, no effect was found in these studies on osteoblaststic cells (unpublished data). These findings are consistent with a non-oxidative stress mechanism associated with the apoptotic process. 
In order to investigate the possible mechanism of action of this vanadium complex, we assessed the signaling transduction pathway of protein phosphorylation. The ability of vanadium to inhibit different phosphatases is well known, as is the importance of protein phosphorylation in the process of osteoblastic differentiation (Xiao et al., 2002). For this reason, we examined the inhibitory potency of VOAsc on PTPases (neutral and alkaline activities) obtained from an UMR106 osteoblastic extract. VOAsc dose-dependently inhibited both phosphatases, with a half maximum effect observed around $10 \mu \mathrm{M}$. These results were consistent with VOAsc inhibitory potency on ALP activity in UMR106 osteoblasts. These findings suggest that this compound could be acting by regulation of intracellular phosphorylation levels.

In consequence, ERK activation/phosphorylation was then investigated as a possible target for VOAsc action. This compound was found to enhance P-ERK in a time-dependent manner in osteoblasts. First, P-ERK was increased after $30-60 \mathrm{~min}$ incubation as observed by Western immunoblot using a phospho-specific antibody. Second, the translocation of P-ERK was shown to occur from the cytoplasm to the nucleus after incubation with $1 \mathrm{mM}$ VOAsc, with a similar time course. Finally, the VOAsc stimulated osteoblastic proliferation and differentiation were partially or totally blocked by PD 98058 or UO126, both inhibitors of MAPK pathway. In addition, other signal transduction mechanisms seem to be important in the osteogenic action of this compound, such as the PI-3-K pathway and calcium uptake, since wortmanin and the L-type calcium channel blocker nifedipine partly inhibited the effects of VOAsc.

One important question about the bioactivity of vanadium complexes is related to their stability in solution. In this context, we have performed a series of stability studies and found that under our experimental conditions VOAsc solutions remained stable during the period of sample preparation and addition to culture media. Nevertheless, these results are only an approach to the real phenomenon. For example, the cell will take up the vanadium complex very soon after it is added to the culture media. We have previously performed NMR and EPR studies with osteoblasts in culture and observed that the vanadyl(IV) uptake occurred after $1 \mathrm{~h}$ incubation, and no vanadium $(\mathrm{V})$ could be observed in the supernatant or inside the cells during the next $4 \mathrm{~h}$ (unpublished observations). Thus, in general it can be assumed that vanadium compounds interact with the cells and generate signaling that subsequently induces biological effects in the term of minutes. The stability of this VOAsc complex is not surprising, since recently Sakurai et al. (2004) showed that ascorbic acid partially stabilized a solution of vanadyl as measured by EPR signal during $6 \mathrm{~h}$. Altogether, these observations suggest that under our working conditions the VOAsc complex solution is relatively stable.

In conclusion, the vanadium(IV)-ascorbic acid complex stimulates osteoblastic differentiation and mineralization in a model of osteoblasts in culture, suggesting an osteogenic activity for this compound. The mechanism of action of this complex seems to involve the inhibition of several phosphatases and activation of the ERK pathway. In addition, calcium and PI-3-K could also be involved in the osteogenic potential of VOAsc. Further in vitro and in vivo experiments need to be performed in order to assess the possible use of VOAsc as a pharmacological tool for bone tissue regeneration.

\section{Acknowledgements}

This study was partially supported by grants from Universidad Nacional de La Plata and CICPBA. DAB is a fellow of CICPBA, MSM is a fellow of CONICET and $A M C$ is a member of the Carrera del Investigador, CICPBA.

\section{References}

Barrio, D. A., Braziunas, M. D., Etcheverry, S. B., \& Cortizo, A. M. (1997). Maltol complexes of vanadium(IV) and (V) regulate in vitro alkaline phosphatase activity and osteoblast-like cell growth. Journal of Trace Element in Medicine and Biology, 11, 110-115.

Barrio, D. A., Williams, P. A., Cortizo, A. M., \& Etcheverry, S. B. (2003). Synthesis of a new vanadyl(IV) complex with trehalose (TreVO): Insulin-mimetic activities in osteoblast-like cells in culture. Journal of Biological Inorganic Chemistry, 8, 459-468.

Cortizo, A. M., Bruzzone, L., Molinuevo, S., \& Etcheverry, S. B. (2000). A possible role of oxidative stress in the vanadium-induced cytotoxicity in the MC3T3E1 osteoblast and UMR106 osteosarcoma cell lines. Toxicology, 147, 89-99.

Domingo, J. L. (2002). Vanadium and tungsten derivatives as antidiabetic agents: A review of their toxic effects. Biological Trace Element Research, 88, 97-112.

Etcheverry, S. B., Barrio, D. A., Cortizo, A. M., \& Williams, P. A. (2002). Three new vanadyl(IV) complexes with non-steroidal antiinflammatory drugs (Ibuprofen, Naproxen and Tolmetin). Bioactivity on osteoblast-like cells in culture. Journal of Inorganic Biochemistry, 88, 94-100.

Etcheverry, S. B., Williams, P. A., Salice, V. C., Barrio, D. A., Ferrer, E. G., \& Cortizo, A. M. (2002). Biochemical properties and mechanism of action of a vanadyl(IV)-aspirin complex on bone cell lines in culture. Biometals, 15, 37-49.

Ferrer, E. G., Williams, P. A. M., \& Baran, E. J. (1998). Interaction of the vanadyl(IV) cation with L-ascorbic acid and related systems. Zeitschrift fur Naturforsch, 53b, 256-262.

Kousteni, S., Chen, J. R., Bellido, T., Han, L., Ali, A. A., O’Brien, C. A., et al. (2002). Reversal of bone loss in mice by nongenotropic signaling of sex steroids. Science, 298, 843-846. 
Laemmli, U. K., Beguin, F., \& Gujer-Kellenberger, G. (1979). A factor preventing the major head protein of bacteriophage T4 from random aggregation. Journal of Molecular Biology, 47, 69-85.

Lai, C. F., Chaudhary, L., Fausto, A., Halstead, L. R., Ory, D. S., Avioli, L. V., et al. (2001). Erk is essential for growth, differentiation, integrin expression, and cell function in human osteoblastic cells. Journal of Biological Chemistry, 276, 14443-14450.

Lowry, O. H., Rosebrough, N. J., Farr, A. L., \& Randall, R. J. (1951). Protein measurement with the Folin phenol reagent. Journal of Biological Chemistry, 193, 265-275.

Manolagas, S. C. (2000). Birth and death of bone cells: Basic regulatory mechanisms and implications for the pathogenesis and treatment of osteoporosis. Endocrine Reviews, 21, 115-137.

McCarthy, A. D., Etcheverry, S. B., Bruzzone, L., \& Cortizo, A. M. (1997). Effects of advanced glycation end-products on the proliferation and differentiation of osteoblast-like cells. Molecular and Cellular Biochemistry, 170, 43-51.

Molinuevo, M. S., Barrio, D. A., Cortizo, A. M., \& Etcheverry, S. B. (2004). Antitumoral properties of two new vanadyl(IV) complexes on osteoblasts in culture. Role of apoptosis and oxidative stress. Cancer Chemotherapy and Pharmacology, 53, 163-172.

Park, S., Han, S. S., Park, C. H., Hahm, E. R., Lee, S. J., Park, H. K., et al. (2004). L-Ascorbic acid induces apoptosis in acute myeloid leukemia cells via hydrogen peroxide-mediated mechanisms. International Journal of Biochemistry \& Cell Biology, 36, 2180-2195.

Partridge, N. C., Alcorn, D., Michelangeli, V. P., Ryan, G., \& Martin, T. J. (1983). Morphological and biochemical characterization of four clonal osteogenic sarcoma cell lines of rat origin. Cancer Research, 43, 4308-4314.

Peters, K. G., Davis, M. G., Howard, B. W., Pokross, M., Rastogi, V., Diven, C., et al. (2003). Mechanism of insulin sensitization by BMOV (bis maltolato oxo vanadium); unliganded vanadium $\left(\mathrm{VO}_{4}\right)$ as the active component. Journal of Inorganic Biochemistry, 96, 321-330

Plotkin, L. I., Weinstein, R. S., Parfitt, A. M., Roberson, P. K., Manolagas, S. C., \& Bellido, T. (1999). Prevention of osteocyte and osteoblast apoptosis by bisphosphonates and calcitonin. Journal of Clinical Investigation, 104, 1363-1374.

Quarles, L. D., Yohay, D. A., Lever, L. W., Caton, R., \& Wenstrup, R. J. (1992). Distinct proliferative and differentiated stages of murine MC3T3-E1 cells in culture: An in vitro model of osteoblast development. Journal of Bone and Mineral Research, 7, 683-692.

Sakurai, H., Onohara, T., Adachi, Y., Kawabe, K., Yasui, H., \& Takada, J. (2004). A news candidate for insulinomimetic vanadium complex: Synergism of oxovanadium(IV)porphyrin and sodium ascorbate. Bioorganic \& Medicinal Chemistry Letters, 14, 1093 1096.
Salgado, A. J., Coutinho, O. P., \& Reis, R. L. (2004). Bone tissue engineering: State of the art and future trends. Macromolecular Bioscience, 4, 643-765.

Shechter, Y., Li, J., Meyerovitch, J., Gefel, D., Bruck, R., Elberg, G., et al. (1995). Insulin-like actions of vanadate are mediated in an insulin-receptor-independent manner via non-receptor protein tyrosine kinases and protein phosphotyrosine phosphatases. Molecular and Cell Biochemistry, 153, 39-47.

Srivastava, A. K., \& Mehdi, M. Z. (2005). Insulino-mimetic and antidiabetic effects of vanadium compounds. Diabetes Medicine, 22, $2-13$.

Steller, H. (1995). Mechanisms and genes of cellular suicide. Science, 267, 1445-1449.

Thompson, K. H., \& Orvig, C. (2004). Vanadium compounds in the treatment of diabetes. Metal Ions in Biological Systems, 41, 221-252.

Tracey, A. S., \& Gresser, M. J. (1986). Interaction of vanadate with phenol and tyrosine: Implications for the effects of vanadate on systems regulated by tyrosine phosphorylation. Proceedings of the National Academy of Sciences of the United States of America, 83, 609-613.

Tuan, R. S. (2004). Biology of developmental and regenerative skeletogenesis. Clinical Orthopaedics and Related Research, 427(Suppl.), S105-S117.

Tullberg-Reinert, H., \& Jundt, G. (1999). In situ measurement of collagen synthesis by human bone cells with a Sirius red-based colorimetric microassay: Effects of transforming growth factor beta2 and ascorbic acid 2-phosphate. Histochemistry and Cell Biology, 112, 271-276.

Ueno, A., Kitase, Y., Moriyama, K., \& Inoue, H. (2001). MC3T3E1conditioned médium-induced mineralization by clonal rat dental pulp cells. Matrix Biology, 20, 347-355.

Williams, P. A. M., Molinuevo, M. S., Okulik, N., Jubert, A. H., \& Etcheverry, S. B. (2005). Synthesis, characterization and biological properties of vanadyl(IV) complexes of diclofenac and indomethacin: An experimental and theoretical study. Applied Organometallic Chemistry, 19, 711-718.

Xiao, G., Gopalakrishnan, R., Jiang, D., Reith, E., Benson, M. D., \& Franceschi, R. T. (2002). Bone morphogenetic proteins, extracellular matrix, and mitogen-activated protein kinase signaling pathways are required for osteoblast-specific gene expression and differentiation in MC3T3-E1 cells. Journal of Bone and Mineral Research, 17, 101-110.

Xiao, G., Jiang, D., Gopalakrishnan, R., \& Franceschi, R. T. (2000). Fibroblast growth factor 2 induction of the osteocalcin gene requires MAPK activity and phosphorylation of the osteoblast transcription factor, Cbfa1/Runx2. Journal of Biological Chemistry, 277, 36181-36187. 